\title{
Integración o inclusión: El dilema educativo en la atención a la diversidad
}

\author{
José Domínguez Alonso', Antonio López Castedoii, \\ Margarita Rosa Pino Justeiii \& Elia Vázquez Varelaiv \\ Universidade de Vigo, Espanha
}

\begin{abstract}
Resumen
El sistema educativo español aboga por la inclusión educativa en la atención al alumnado diverso en los centros de enseñanza obligatoria. Se realizó una investigación cualitativa-cuantitativa, cuyo objetivo era comprobar la sustitución paulatina de la perspectiva integradora por las prácticas inclusivas en los centros educativos. Así, los métodos se ejecutaron de manera secuencial: en una primera etapa se recolectaran y analizaran los datos cualitativos (grupos de enfoque), y en una segunda fase se recabaran y examinaran datos cuantitativos (cuestionario de opinión). El primer análisis muestra un predominio de aspectos que entorpecen la atención a la diversidad (64\%), frente a los que la favorecen (36\%). El segundo refleja un predominio del modelo de integración (74\%), en detrimento del modelo de inclusión (26\%). Además, el análisis inferencial revela que a mayor experiencia docente menor importancia se le concede a la atención del alumnado diverso, y viceversa.
\end{abstract}

Palabras clave

Educación inclusiva; Integración; Atención a la diversidad

\section{Introducción}

Después de muchos años de que se pusiera en marcha la respuesta a las diferencias individuales como una segunda red del sistema educativo 
(educación diferencial, educación especial, educación compensatoria) apoyada en un proyecto integrador de una audacia insospechada pero con unos resultados muy cuestionados, es el momento (entrada en vigor y puesta en funcionamiento de la nueva Ley Orgánica para la Mejora de la Calidad Educativa: LOMCE, 8/2013) de proclamar la necesidad de apuntalar el reto de la inclusión.

Paralelamente, en Portugal, desde el punto de vista legislativo se han facilitado condiciones para que todo el alumnado aun con problemáticas complejas pueda integrarse en la escuela ordinaria (Silva, 2009). Además de otras modalidades específicas, es importante reseñar la DL 3/08, de 7/01, similar a la DL 319/91, de 23/08, que fue derogada, donde se definen las medidas educativas para estos estudiantes: apoyo pedagógico personalizado, adaptaciones curriculares individualizadas, ajustes en el proceso de matrícula y evaluación, tecnologías de apoyo y un curriculum específico individualizado, medidas similares a otros países de la Unión Europea.

Cierto es que todas las transformaciones en la atención del alumnado con dificultades de aprendizaje han sido fruto de los cambios experimentados en la educación general, por lo que su mayor presencia en los contextos educativos ordinarios nos lleve a innovar prácticas y metodologías en sintonía con los valores inclusivos (Echeita, 2008), muy refrendados a nivel teórico pero con escasa aplicación en la práctica diaria de los centros de enseñanza.

En el marco de las actuales políticas educativas europeas (UNESCO, 2004), existe el reconocimiento de la educación inclusiva como el soporte fundamental que asegura la igualdad de oportunidades para las personas con necesidades específicas en todos los aspectos de sus vidas (educación, formación, empleo y vida social). En esta misma línea, España aboga por una trayectoria escolar inclusiva asegurando el reconocimiento de la diversidad y la interculturalidad. Así pues, todo el alumnado debe estar escolarizado en los centros ordinarios, recibiendo la educación más acorde a sus características, de forma que los apoyos, el recibimiento y la calidad de la enseñanza llegue a todos los estudiantes sin excepciones (Arnaiz, 2003). No obstante, lograr una oferta común para todo el alumnado y al mismo tiempo respuestas diferenciadas para cada uno de ellos, es decir, una atención igual y, a la par, diferente para todos los estudiantes en el actual sistema educativo, sigue teniendo muchas dificultades, reticencias y restricciones (Ainscow, 2007; 
Dyson \& Gallannaugh, 2007; Florian, 2005; Low, 2007). La escuela arrastra, a lo largo de su historia, un nexo segregador lo suficientemente robusto, efectivo y cercano que impide velar por el necesario mantenimiento del más profundo e irrenunciable principio de la enseñanza inclusiva: "la atención a la diversidad no puede ser algo que hacemos para unos pocos, sino un principio esencial que debe regir la acción escolar con el conjunto del alumnado" (González Manjón, 2013, p. 105).

La evolución de la atención al alumnado diverso pasa de la desatención y la marginación inicial a la conocida "educación especial" o "educación compensatoria", y visto la segregación que ésta generaba dio lugar a la normalización e integración de las personas en un ambiente menos restrictivo, lo que finalmente debe derivar en la inclusión educativa, laboral y social de los individuos, haciendo interactuar a todos los intervinientes y los propios contextos (Sanches, 2011; Silva, 2009; Verdugo, 2003). No obstante, la integración ha sido y continúa siendo uno de los fenómenos de mayor trascendencia para el alumnado con problemas de aprendizaje en el campo educativo, haciendo incuestionable el derecho de todas las personas a la escolarización, siempre que sea posible, en los centros ordinarios, lo que está dificultando enormemente la aceptación plena de la inclusión. Llegado a este punto, nos encontramos en un momento de tensión entre dos conceptos, integración e inclusión, generado por la continua evolución del campo de la diversidad.

En el proceso integrador, lo relevante es que traslada el enfoque individualizado y rehabilitador, propio de la educación especial (escuelas segregadas) al contexto de la escuela regular, haciendo ajustes y adaptaciones solamente para el alumnado considerado de necesidades educativas específicas y no para todos los alumnos del centro. Desde esta perspectiva, aún hoy, muchos equiparan la educación con la asistencia a un centro escolar (Vega, 2007), creando así una segunda red de enseñanza (especial) que nunca se llegó a eliminar, sino que sigue fortaleciéndose con la denominada "red de apoyo" (Núñez, 2008) que incorpora a un nuevo alumnado con necesidades educativas no asociadas a discapacidades (deprivación social, inmigración, retraso escolar,...). La experiencia integradora fue, y sigue siendo, muy controvertida y problemática debido a que contribuyó más a mantener las situaciones formales de ubicación en 
aulas ordinarias del alumnado con necesidades educativas específicas que a desenvolver una escuela capaz de acoger a todo el alumnado (Vlachou, 1999). Esta situación lleva a Ainscow (1999) a sostener que "se ha utilizado la palabra integración para describir procesos mediante los cuales ciertos niños reciben apoyos con el fin de que puedan participar en los programas existentes (en gran medida sin modificaciones) de los colegios" (p. 25).

Ahora bien, si el objetivo principal de la integración era conseguir que el alumnado con dificultades de aprendizaje formara parte de un único sistema general de enseñanza (Sanches \& Teodoro, 2006), lo que consiguió fue el efecto contrario, condenándola a ser una medida de asentamiento físico, pues se pensaba y asumía la idea de una educación distinta, didácticamente diferente, con un currículo singular y segregado, y con unos programas de desarrollo individual construidos a partir de ese currículo paralelo. Esta situación y el nuevo contexto socioeducativo emergente hace surgir un nuevo término: inclusión. En primer lugar, para paliar las situaciones de desintegración y exclusión a las que se están viendo sometidos muchos alumnos bajo la bandera de la integración; en segundo lugar, como un intento de reconstruir el enfoque deficitario individualista y médico dominante; $y$, en tercer lugar, como una reivindicación de que todos los alumnos con o sin necesidades educativas especiales reciban una educación de calidad en las aulas regulares (Arnaiz, 2003).

Es importante, pues, una profunda transformación del sistema de enseñanza que haga un planteamiento de la educación desde una perspectiva de escuela inclusiva, considerada como una filosofía y práctica educativa emergente que pretende mejorar el acceso a un aprendizaje de calidad en las clases ordinarias para todos los estudiantes, a través de contextos de aprendizaje inclusivos desarrollados desde el marco de un currículo común (Ainscow \& Miles, 2008; Marchesi, 2000; Parrilla, 2001). En consecuencia, estableciendo que cada comunidad educativa debe definir y concretar el término inclusión en función de su historia, contexto, cultura, y sobre todo sus condicionantes económicos, políticos... (Nilholm, 2006), y sin la pretensión de hacer un acopio exhaustivo de las diversas concepciones sobre la educación inclusiva surgidas en este nuevo periodo de atención al alumnado con necesidades educativas específicas; se recoge, únicamente a título ilustrativo, algunas de las más interesantes (tabla 1). 
Tabla 1 - Selección de algunas definiciones de inclusión

\begin{tabular}{|c|c|}
\hline AUTORES & CONCEPTO \\
\hline Echeita, 2008, p. 13 & $\begin{array}{l}\text { "La inclusión debe verse como un proceso de reestructuración escolar relativo a la } \\
\text { puesta en marcha, precisamente, de procesos de innovación y mejora que acerquen a } \\
\text { los centros al objetivo de promover la presencia, la participación y el rendimiento de } \\
\text { todos los estudiantes de su localidad - incluidos aquellos más vulnerables a los } \\
\text { procesos de exclusión -, aprendiendo de esa forma a vivir con la diferencia y a mejorar } \\
\text { gracias, precisamente, a esas mismas diferencias entre el alumnado" }\end{array}$ \\
\hline Parrilla, 2009, p. 104 & $\begin{array}{l}\text { "Es un concepto que tiene su razón de ser en la existencia de situaciones y procesos } \\
\text { de exclusión" }\end{array}$ \\
\hline INICO, 2009 , p. 20 & $\begin{array}{l}\text { "El concepto de un cambio de paradigma de alto nivel para los sistemas educativos a } \\
\text { fin de incluir y servir a los niños de manera efectiva; y el mandato específico para que } \\
\text { los alumnos con discapacidad asistan a las escuelas ordinarias y a las aulas con sus } \\
\text { hermanos y compañeros sin discapacidad, con los apoyos que necesitan para tener } \\
\text { éxito" }\end{array}$ \\
\hline Moriña, 2011, p. 200 & $\begin{array}{l}\text { "La educación inclusiva ha sido definida como un proceso que persigue responder a } \\
\text { las necesidades de todo el alumnado y satisfacerlas mediante una mayor participación } \\
\text { en el aprendizaje, las culturas y las comunidades" }\end{array}$ \\
\hline Blanco, 2014, p. 12 & $\begin{array}{l}\text { "El concepto de inclusión o de educación inclusiva suele asociarse a los estudiantes } \\
\text { con discapacidad o con necesidades educativas especiales, o a quienes viven en } \\
\text { contextos de pobreza, aunque progresivamente se está adoptando un enfoque más } \\
\text { amplio concibiéndola como un medio para lograr un acceso equitativo a una educación } \\
\text { de calidad, sin ningún tipo de discriminación" }\end{array}$ \\
\hline
\end{tabular}

De esta forma, en la escuela inclusiva no tienen cabida los currículos paralelos; es la clase en su conjunto, el centro en su totalidad, el que adopta una nueva forma organizativa de respuesta a todos sus alumnos, centrada más en buscar formas alternativas válidas para todos que en responder a un déficit de un determinado estudiante (Domínguez \& Pino, 2007). En efecto, el concepto de inclusión implica modificar substancialmente la estructura, funcionamiento y propuestas pedagógicas de las escuelas para dar respuesta a las necesidades educativas de todos y cada uno de sus alumnos, de manera que todos tengan éxito en su proceso de aprendizaje y participen en igualdad de condiciones. Lo difícil y complicado es saber cómo implementar en la práctica diaria de los centros educativos los ideales de la enseñanza inclusiva. A este respecto, la Agencia Europea para el Desarrollo de la Educación Especial (European Agency for Development in Special Needs Education, 2003, 2006) señala siete factores decisivos para una práctica de inclusión educativa efectiva en las aulas (tabla 2).

En las últimas dos décadas, la dinámica educativa intercultural favoreció más la construcción de una sociedad sensible con las desigualdades y una tendencia creciente a la incorporación al discurso y la reflexión del concepto de exclusión y de cohesión social, como una forma renovada de afrontar la desigualdad y la educación compensatoria. En este sentido, todos los indicadores, tanto ideológicos como socioeconómicos, 
hablan de una evolución social en los países occidentales que conduce a unas sociedades con índices de desigualdad crecientes (Essomba, 2003). La lucha contra la exclusión social requiere un reconocimiento de la diversidad que se transforme en medidas en el plano jurídico-político, en el compromiso moral, en el desarrollo de una ética común y en el papel fundamental que debe desarrollar la educación (García, 2005).

\section{Tabla 2 - Factores decisivos para las prácticas inclusivas en centros educativos}

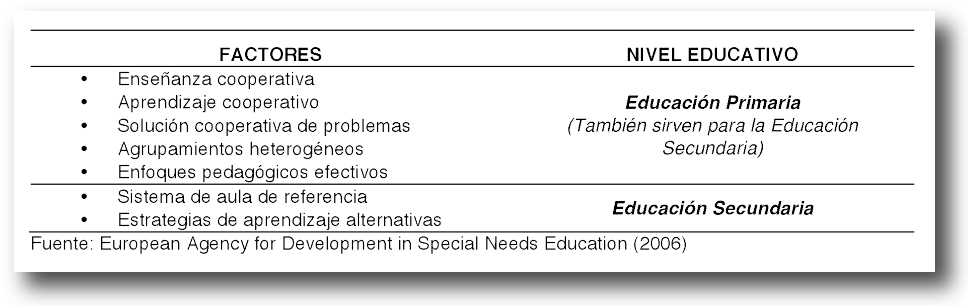

Finalmente, la visión que nos ofrecen los estudios sobre prácticas integradoras o inclusivas en la última década aborda una gran variedad de cuestiones relativas al reconocimiento expreso de la heterogeneidad y el derecho de todo el alumnado a recibir una educación adaptada a su individualidad en entornos ordinarios de enseñanza-aprendizaje. Así pues, entre las áreas de investigación que han recibido una mayor atención se encuentran: análisis críticos sobre el lenguaje y presupuestos que acompañan al discurso de la integración e inclusión (Booth, Ainscow, Black-Hawkins, Vaughn, \& Shaw, 2000; Silva, 2009); el fenómeno de la díada inclusiónexclusión (Escudero, 2005; Escudero, González, \& Martínez, 2009; Jiménez, Luengo, \& Tabernero, 2009); los efectos de la integración-inclusión en el alumnado y sus prácticas (Arnaiz, 2012; Fernández, 2010; Martínez, 2005; Moliner, Sales, Ferrández, Moliner, \& Roig, 2012); y, sobre todo, las actitudes y formación del profesorado acerca de la filosofía inclusiva (Fullan, 2007; Khochen \& Radford, 2012; Kim, 2011; Shevlin, Winter, \& Flynn, 2013; Silva, 2003;).

Siendo conscientes que la ciudadanía inclusiva fomenta "el respeto y la comprensión de las diferencias" (Pérez \& Sarrate, 2013, p. 100), el objetivo 
de este estudio es doble: de una parte comprobar si el profesorado es consciente de las diferencias en el discurso teórico que acompaña a los conceptos de integración e inclusión; y, de otra, identificar las prácticas o acciones sobre las que se asienta la atención del alumnado diverso en los centros educativos.

\section{Material y métodos}

Para dar respuesta al objetivo formulado y comprobar e identificar el predominio de la integración frente a la inclusión en los centros educativos, se destacan a continuación los aspectos de mayor relevancia en el estudio: las muestras, los instrumentos o procedimientos en la recogida de datos, así como las técnicas estadísticas utilizadas. Para ello, se utiliza un diseño exploratorio secuencial (DEXPLOS), con dos fases complementarias pero diferenciadas (Walmsley, 2004): la primera de corte cualitativo desde dentro de los centros educativos (investigación inclusiva), y la segunda (investigación sobre inclusión) a través de la opinión de los jefes de departamentos de orientación (metodología cuantitativa).

\section{Participantes}

La primera fase longitudinal de corte cualitativo se desarrolló a través de grupos de enfoque (focus group) durante cinco cursos académicos (periodo 2006-2011) en cuatro centros educativos. En total han existido cinco grupos de enfoque y han participado treinta profesores, dos asesores del centro de formación y recursos, cuatro directores, cinco jefes de departamento de orientación educativa y un único coordinador (experto en dinámicas de grupo).

En la parte transversal cuantitativa participaron 140 jefes de los departamentos de orientación de educación primaria en la Comunidad Autónoma de Galicia ( $\mathrm{N}=229$ ), lo que representa un $61.14 \%$ de la población total. Así pues, la muestra estuvo compuesta por orientadores con edades comprendidas entre los 30 y los 60 anos, con unos porcentajes del $44.5 \%$ (tramo de 41 a 50 años) y un $24.5 \%$ (tramo de 30 a 40 años). Además se tuvo presente el género (Hombres $=22.7 \%$, Mujeres $=66.4 \%$ ); la experiencia educativa (entre 11 y 18 años $=24.7 \%$, más de 18 años $=53.6 \%$ ); y la 
formación (Diplomados $=99.3 \%$, de los cuales un $64.8 \%$ también son licenciados).

\section{Procedimiento}

Como punto de partida, se realizaron revisiones documentales y bibliográficas (nacionales e internacionales), las descripciones y aportaciones de los grupos de enfoque creados en los propios centros educativos; finalmente, se introduce la abundante información obtenida en el programa informático Aquad 4.0. De esta forma se consiguió una radiografía razonable y real de las posibles dimensiones e indicadores de las prácticas integradoras o inclusivas en los centros de enseñanza. Partiendo del enfoque holístico obtenido en el análisis cualitativo, se procede a la elaboración de un cuestionario de opinión utilizando una técnica cualitativa de previsión grupal que se nutre del juicio de expertos (método Delphi) y un estudio piloto. El cuestionario final (fiabilizado y validado), denominado CUADIVER (Cuestionario de Atención a la Diversidad), fue enviado por correo electrónico a los centros educativos. Los análisis de datos se han realizado con ayuda del paquete estadístico SPSS 21.

\section{Instrumentos}

Como se ha comentado, la investigación implicó: inmersión inicial en el campo, observación abierta y observación particularizada. La parte cualitativa se realizó a través de grupos de enfoque en los propios centros educativos, con cinco sesiones en cada curso académico (25 en total). Para elaborar, optimizar y complementar la discusión se diseña una matriz común de recogida de datos en función del objetivo de investigación, realizando una prueba piloto para asegurar su pertinencia (Cuevas, 2013).

Asimismo, se ha conocido la opinión de los orientadores escolares a través del cuestionario CUADIVER. El instrumento ofrece una escala en la que las personas encuestadas deben responder, a través de una escala Likert, sobre su estimación del grado de acuerdo (Mucho) o desacuerdo (Nada). La escala responde a una serie de ítems o indicadores que representan las actuaciones en la atención a la diversidad desde los centros educativos. Ofrece un a de Cronbach para el conjunto del cuestionario de .93 
y de .87 para el factor "actuaciones en la atención a la diversidad" (Pino, Domínguez, \& López, 2007).

\section{Resultados}

Los indicadores que se proponen para la valoración de la integración o inclusión escolar en los centros educativos están en relación con las percepciones y opiniones sobre la situación real de la atención al alumnado diverso en los centros educativos de primaria. Se presentan los resultados ofreciendo en primer lugar los datos descriptivos, dejando para una reflexión final la discusión sobre posibles causas e inferencias que de ellos se puedan derivar.

\section{Análisis cualitativo}

Del contenido emergente obtenido en los grupos de enfoque (analizado a través del paquete informático Aquad 4.0) sobre la realidad de los centros educativos, se observa que los aspectos desfavorables $(63.8 \%)$ son superiores a los favorables (36.2\%) en la atención a la diversidad. Entre los aspectos favorables cabe señalar que conceden gran importancia y muestran preocupación hacia la atención del alumnado con necesidades educativas específicas, destacando la necesidad de su inclusión dentro del grupo clase, además de considerar positiva la incorporación de las nuevas tecnologías de la información y la comunicación en el proceso de enseñanza-aprendizaje de este alumnado. Sin embargo, en los desfavorables destacan la escasa formación, el tiempo y la actitud del profesorado ante esta temática, la falta de trabajo colaborativo y la tendencia a preferir grupos homogéneos, así como la falta de recursos humanos y materiales. También denuncian el rol pasivo de la administración en el proceso de inclusión y el diseño de un sistema educativo único que incluya a todo el alumnado sin excepciones.

\section{Análisis descriptivo}

Del análisis descriptivo (tabla 3) se observan dos ámbitos de actuación muy marcados: las intervenciones en el proceso de enseñanza-aprendizaje de todo el alumnado (tendencia más inclusiva) y las realizadas exclusivamente con el alumnado con dificultades de aprendizaje (tendencia 
más integradora). De manera detallada, acudiendo a los porcentajes percibidos de aparición de cada tarea, se observa que las más realizadas desde los departamentos de orientación son: evaluaciones psicopedagógicas del alumnado con necesidades educativas específicas (79.2\%); la elaboración de informes psicopedagógicos (78.1\%); la colaboración y el asesoramiento en las medidas que favorecen los procesos de aprendizaje del alumnado con dificultades de aprendizaje: refuerzos educativos y adaptaciones curriculares (74.5\%); y proporcionar información profesional o académica (63.6\%).

Por el contrario, entre las menos desarrolladas se encuentran: los planes de acogida al alumnado nuevo del centro (49.1\%); la participación y colaboración en los proyectos del centro (45.7\%); proporcionar materiales, criterios metodológicos y de evaluación en los procesos de enseñanza (39.6\%); marcar pautas de actuación para el alumnado con dificultades en mejora de la diversidad (36.4\%); establecer medidas de prevención generales en el centro para favorecer la diversidad del alumnado (23.6\%), información, colaboración y elaboración de programas para las familias (17.1\%); y, en último lugar, el facilitar a los tutores pautas de observación relacionadas con los contenidos, procedimientos y organización del aula (10.4\%).

Tabla 3 - Actuaciones para atender a la diversidad desde los Departamentos de Orientación

\begin{tabular}{|c|c|c|c|}
\hline \multirow[b]{2}{*}{ ACTIVIDADES O TAREAS QUE REALIZAN } & \multicolumn{3}{|c|}{ PORCENTAJES \% } \\
\hline & $\begin{array}{l}\text { Nada/ } \\
\text { Poco }\end{array}$ & Suficiente & $\begin{array}{c}\text { Bastante/ } \\
\text { Mucho }\end{array}$ \\
\hline $\begin{array}{l}\text { 1. Evaluación psicopedagógica del alumnado con necesidades } \\
\text { educativas específicas }\end{array}$ & 6.6 & 14.2 & 79.2 \\
\hline $\begin{array}{l}\text { 2. Colaborar y asesorar en las medidas que favorecen los } \\
\text { procesos de aprendizaje del alumnado con dificultades de } \\
\text { aprendizaje: refuerzo educativo y adaptaciones curriculares }\end{array}$ & 7.5 & 17.9 & 74.5 \\
\hline $\begin{array}{l}\text { 3. Proporcionar materiales, criterios metodológicos y de } \\
\text { evaluación en los procesos de enseñanza }\end{array}$ & 26.4 & 34.0 & 39.6 \\
\hline $\begin{array}{l}\text { 4. Facilitar a los tutores pautas de observación relacionadas con } \\
\text { los contenidos, procedimientos y organización del aula }\end{array}$ & 62.3 & 27.4 & 10.4 \\
\hline $\begin{array}{l}\text { 5. Información, colaboración y elaboración de programas para } \\
\text { las familias }\end{array}$ & 52.4 & 30.5 & 17.1 \\
\hline $\begin{array}{l}\text { 6. Participar y colaborar en los proyectos del centro: Proyecto } \\
\text { Educativo (PEC),... }\end{array}$ & 23.8 & 30.5 & 45.7 \\
\hline 7. Elaborar informes psicopedagógicos & 9.6 & 12.3 & 78.1 \\
\hline 8. Proporcionar información profesional o académica & 9.1 & 26.4 & 63.6 \\
\hline $\begin{array}{l}\text { 9. Marcar pautas de actuación para el alumnado con dificultades } \\
\text { en mejora de la diversidad }\end{array}$ & 31.7 & 31.8 & 36.4 \\
\hline 10. Aplicación de pruebas: test, escalas... & 13.8 & 34.5 & 51.6 \\
\hline 11. Planes de acogida al alumnado nuevo del centro & 19.0 & 31.8 & 49.1 \\
\hline $\begin{array}{l}\text { 12. Establecer medidas de prevención generales en el centro } \\
\text { para favorecer la diversidad del alumnado }\end{array}$ & 42.7 & 33.6 & 23.6 \\
\hline
\end{tabular}


A partir de estos datos, se infiere que las tareas específicas encaminadas al alumnado con necesidades educativas específicas son más frecuentes en los centros educativos que las dirigidas a todo el alumnado. Por lo tanto, el proceso integrador sigue muy presente en las aulas en detrimento de la inclusión educativa.

\section{Análisis inferencial}

En cuanto a si existen variaciones en las valoraciones por parte de los orientadores en referencia al género, edad, experiencia en los departamentos de orientación o titulación académica, se aprecia que no aparecen diferencias significativas excepto en el caso de la experiencia $[F(4,89)=5.65 ; p<.001]$. Se aprecia, en los contrastes a posteriori (Scheffé), que a mayor experiencia (15-18 años) menor importancia se le concede a la atención de la diversidad, y viceversa (tabla 4).

Tabla 4 - Análisis inferencial de la atención a la diversidad según el género, edad, experiencia y titulación académica

\begin{tabular}{|c|c|c|c|c|}
\hline \multirow{3}{*}{ Género } & $\mathrm{x}$ & $\mathrm{s}$ & $t$ & $\mathrm{p}$ \\
\hline & $\begin{array}{l}47.2 \\
48.7\end{array}$ & $\begin{array}{l}8.3 \\
6.8\end{array}$ & -.718 & .475 \\
\hline & \multicolumn{2}{|c|}{$\mathrm{F}$} & \multicolumn{2}{|c|}{$P$} \\
\hline Edad & \multicolumn{2}{|c|}{.539} & \multicolumn{2}{|c|}{.585} \\
\hline Experiencia & \multirow{2}{*}{\multicolumn{2}{|c|}{$\frac{5.651}{539}$}} & \multirow{2}{*}{\multicolumn{2}{|c|}{.000}} \\
\hline Titulación Académica & & .539 & & \\
\hline
\end{tabular}

\section{Análisis factorial}

Del análisis factorial $(\mathrm{KMO}=.96)$ se desprende un patrón muy similar al encontrado en la parte descriptiva (tabla 5). Muestra la existencia de dos factores que explican un $61.14 \%$ de la varianza de los datos. El primer factor obtiene un mayor porcentaje de la varianza explicada (49.76\%). Está formado por la intervención y prevención sobre el alumnado con dificultades de aprendizaje (dimensión integradora). Los pesos factoriales correspondientes a los ítems de este factor oscilan entre .49 y .88, siendo su coeficiente de fiabilidad de .88. El segundo factor explica un $11.38 \%$ de la varianza, correspondiendo a las actuaciones encaminadas a todo el alumnado (enfoque 
de inclusión). Sus pesos factoriales se sitúan entre .57 y .77, con una fiabilidad de .82 .

Tabla 5 - Análisis factorial de las actuaciones en la atención a la diversidad

\begin{tabular}{|c|c|c|c|}
\hline \multirow[t]{2}{*}{ VARIABLES } & \multicolumn{3}{|c|}{ FACTORES Y VARIABLES QUE EXPLICAN (\%) } \\
\hline & FACTOR 1 & FACTOR 2 & DIMENSIONES \\
\hline Item 3 & 877 & & \\
\hline Item 1 & .862 & & \\
\hline Item 5 & .824 & & Actuaciones específicas en el proceso \\
\hline Item 4 & .776 & & de enseñanza-aprendizaje \\
\hline Item 2 & .592 & & \\
\hline Item 7 & 490 & & \\
\hline Item 11 & & .772 & \\
\hline Item 8 & & .716 & \\
\hline Item 10 & & .664 & - \\
\hline Item 6 & & .661 & the \\
\hline Item 9 & & .573 & \\
\hline Item 12 & & .570 & \\
\hline VARIANZA & 5.97 & 1.37 & \% ACUMULADO DE VARIANZA \\
\hline$\%$ VARIANZA & 49.76 & 11.38 & 61.14 \\
\hline
\end{tabular}

\section{Discusión y conclusiones}

Si bien el armazón del sistema de enseñanza español permanentemente inalterable en el tiempo se nutre de unos preceptos que han presidido una doble vía educativa (ordinaria y especial) de la que no podemos sentirnos orgullosos (remítanse a los últimos resultados obtenidos en el informe PISA), también es cierto que tiene la necesidad de sumarse a las corrientes de actualidad y evolución europeas para seguir siendo un órgano de referencia ineludible para la sociedad española. Por tanto, el reto es inminente y debe afrontarse con la ilusión y responsabilidad en unos momentos en los que hay que aplicar una gran dosis de valor e innovación para asumir los riesgos de un proyecto educativo de carácter inclusivo en los difíciles tiempos que nos acompañan. El sistema de enseñanza inclusiva aspira a convertirse en el mejor y más seguro soporte de calidad educativa que anula metodologías antes irrenunciables (homogeneizantes) y se 
convierte en emblema de cambio generacional en los nuevos sistemas de enseñanza-aprendizaje. La convivencia de los dos sistemas educativos diferenciados (especial y ordinario) debe mitigarse a favor de un sistema único de escuela para todos: "la inclusión debe ser considerada como una búsqueda interminable de formas más adecuadas de responder a la diversidad" (Ainscow, 2003, p. 12).

Este estudio pretende ofrecer un diagnóstico inicial de las concepciones y prácticas en la atención del alumnado con dificultades de aprendizaje en los centros educativos. Pone de manifiesto que, pese a los esfuerzos realizados, los sistemas educativos no están logrando avanzar hacia una mayor inclusión, evidenciando la "permanente dificultad en la enseñanza de encontrar un equilibrio entre lo común y lo diverso" (Marchesi, 2014 , p. 39). En este sentido, los resultados obtenidos no resultan muy halagüeños, planteando el gran reto de intervenir en estas realidades para modificar creencias, percepciones, y prácticas estereotipadas encontradas. La investigación educativa aquí presentada constituye una pequeña aportación que puede contribuir a la visualización y sensibilización de la importancia que tiene la atención a la diversidad de todo el alumnado en los centros de enseñanza, que exige una intervención práctica y metodológica decididas y reales. A pesar de los caminos inesperados a los que pueden dirigirse las investigaciones cuando se utilizan técnicas cualitativas, y especialmente los grupos de enfoque, por su peculiar carácter exploratorio, denominado por Renold (2000, p. 310) "la caja de Pandora de la investigación", se intuye que a pesar del creciente interés en lograr la inclusión de todo el alumnado en el grupo-clase, siguen existiendo desacuerdos favorecidos por las arraigadas experiencias integradoras. Al respecto se observa que entre los aspectos desfavorables para promover las prácticas inclusivas se encuentran: la escasa formación del profesorado; el retraso en la incorporación de las nuevas tecnologías de la información; la falta de trabajo colaborativo y recursos (personales y materiales); el rol pasivo de las administraciones; y, sobre todo, mejorar la actitud hacia los grupos heterogéneos (Alquati \& Beatris, 2012; Leitão, 2006; Tavares \& Sanches, 2011).

En este contexto, algunos de los hallazgos del trabajo muestran que el cambio hacia prácticas de inclusión se está iniciando utilizando el mismo 
soporte integrador que ha servido en la misión de acercamiento y cohesión de los dos sistemas educativos. Una de las condiciones para asegurar el éxito de esta perspectiva inclusiva es que ha de ser querida, llevada a cabo con la seriedad, el rigor, la calidad y el compromiso más profundo en una labor de total cohesión y consistencia de un único sistema educativo en su práctica y sus fines, constituyendo un auténtico referente universal de la enseñanza.

Asimismo, los datos cuantitativos obtenidos evidencian también una tendencia integradora muy marcada (evaluaciones psicopedagógicas, informes psicopedagógicos, refuerzos educativos y adaptaciones curriculares individualizas), frente a prácticas inclusivas (planes de acogida, participación y colaboración en los proyectos de centro, programas de participación de las familias en la escuela, pautas innovadoras de organización y planificación del aula). Además, se aprecia, en el análisis inferencial, que a menor experiencia docente, mayor importancia se concede a la inclusión, y viceversa.

Los resultados demuestran que en los centros educativos existen más carencias que situaciones, recursos o actitudes favorables hacia la inclusión. Se aprecia, además, al igual que en la investigación de Biencito, González, García, Sánchez y Madrid (2009), dos tipos de centros: los que identifican las diferencias del alumnado para separarlo, diseñando planes ajustados dirigidos a este grupo identificado; y los que identifican las diferencias para atenderlas educativamente, diseñando planes ajustados a las diferencias individuales de todos y cada uno de los alumnos del aula para ayudarles a conseguir los objetivos a la vez que desarrollan sus aptitudes. Esta situación puede deberse, como apuntan Álvarez et al. (2002), a que la atención a la diversidad desde una perspectiva de inclusión en el sistema educativo, a pesar del empujón recibido por las leyes educativas, sigue considerándose un reto difícil $y$, en ocasiones, contradictorio con algunos valores sociales vigentes en la actualidad.

Evidentemente, si se sigue siendo incapaces de alcanzar el gran reto que es la educación inclusiva (Sanches, 2005), existe el riesgo serio de que pasemos a la historia como la generación que permitió el final de un sistema único de enseñanza para todo el alumnado. Demasiado sacrificio para un sistema educativo en clara tendencia a la baja en niveles de conocimiento y regresión en consideración social. En síntesis, esta investigación parte de experiencias pioneras con el objetivo de hacer accesible la educación a todo 
el alumnado con dificultades de aprendizaje (Gardou \& Develay, 2005), sacando a la luz concepciones reduccionistas y primarias de antiguos conceptos como educación especial, necesidades educativas especiales, programas de desarrollo individual..., todavía presentes en nuestros centros. Esta realidad pretende abrir un camino iniciado e inexplorado que debe poner su meta en la transformación de los contextos escolares en inclusivos, factor clave para la cimentación de una sociedad más justa y democrática.

En este contexto diferenciado y movidos únicamente por la sana ambición y entrega vocacional de servicio y respaldo a la enseñanza, se pretende avanzar en el ámbito educativo del alumnado con dificultades de aprendizaje, caracterizado por el desarrollo de prácticas segregadoras e integradoras aún presentes, y postulando un nuevo sistema educativo de inclusión que unifique las dos redes educativas existentes, ordinaria y especial, en un único espacio de enseñanza-aprendizaje para todo tipo de alumnado.

\section{Referencias}

Ainscow, M. (1999). Tendiéndoles la mano a todos los estudiantes: Algunos retos y oportunidades. Siglo Cero, 30(1), 37-48.

Ainscow, M. (2003). Desarrollo de sistemas inclusivos. Ponencia presentada en el Congreso "La respuesta a las necesidades educativas especiales en una escuela vasca inclusiva". San Sebastián, del 21 al 31 de octubre de 2003. Versión electrónica: http://sid.usual.es/idocs/F8/FD06565/mel_ainscow.pdf

Ainscow, M. (2007). Towards a more inclusive education system: Where next for special schools? In R. Cigman (Ed.), Included or excluded? (pp. 128-140). London: Routledge.

Ainscow, M., \& Miles, S. (2008). Making education for all inclusive: Where next? Prospects, 38(1), 15-34.

Alquati, C., \& Beatris, C. (2012). Desafios da inclusão: Uma proposta para a qualificação de docentes no Ensino Superior via tecnologias digitais. Revista Portuguesa de Educação, 25(2), 263-280.

Álvarez, V., Rodríguez, A., García, E., Gil, J., López, I., Romero, S., ...Correa, J. (2002). La atención a la diversidad en los centros de enseñanza secundaria: Estudio descriptivo en la provincia de Sevilla. Revista de Investigación Educativa, 20(1), 225-245.

Arnaiz, P. (2003). Educación inclusiva: Una escuela para todos. Málaga: Ediciones Aljibe. 
Arnaiz, P. (2012). Escuelas eficaces e inclusivas: Cómo favorecer su desarrollo. Educación Siglo XXI, 30(1), 25-44.

Biencito, C., González, C., García, M., Sánchez, P., \& Madrid, D. (2009). Diseño y propiedades psicométricas del ACACO-EVADIE. Cuestionario para la evaluación de la atención a la diversidad como dimensión educativa en las instituciones escolares. Relieve, 15(1), 1-36.

Blanco, R. (2014). Inclusión educativa en América Latina: Caminos recorridos y por recorrer. In A. Marchesi, R. Blanco, \& L. Hernández (Coords.), Avances y desafíos de la educación inclusiva en Iberoamérica (pp. 11-35). Madrid: OEI.

Booth, T., Ainscow, M., Black-Hawkins, K., Vaughan, M., \& Shaw, L. (2000). Index for inclusion developing learning and participation in schools. Bristol: CSIE.

Cuevas, A. (2013). A investigar se aprende investigando. Revista Marista de Investigación Educativa, 3(4-5), 134-140

Domínguez, J., \& Pino, M. R. (2007). Atención á diversidade: O discurso e a práctica. Eduga, 51, 79-88.

Dyson, A., \& Gallannaugh, F. (2007). Nacional policy and the development of inclusive school practices: A case study. Cambridge Journal of Education, 37(4), 473-488.

Echeita, G. (2008). Inclusión y exclusión educativa - "Voz y quebranto". REICE Revista Electrónica Iberoamericana sobre Calidad, Eficacia y Cambio en Educación, 6(2), 9-18.

Escudero, J. M. (2005). El fracaso escolar. Nuevas formas de exclusión educativa. En J. G. Molina (Coord.), Exclusión social, exclusión educativa (pp. 83-108). Xátiva: Diálogos.

Escudero, J. M., González, M. T., \& Martínez, B. (2009). El fracaso escolar como exclusión educativa: Comprensión, políticas y prácticas. Revista Iberoamericana de Educación, 50, 41-64.

Essomba, M. A. (2003). Els discursos sobre atenció a la diversitat en la comunitat educativa a Catalunya (Tesi Doctoral). Universitat Autònoma de Barcelona, Barcelona.

European Agency for Development in Special Needs Education (2003). Principios fundamentales de la Educación de Necesidades Especiales: Recomendaciones para responsables políticos. Versión electrónica: www.european-agency.org/

European Agency for Development in Special Needs Education (2006). Educación Especial en Europa (Vol. 2). Factores clave en la Educación Post-Primaria. Versión electrónica: www.european-agency.org/

Fernández, J. M. (2010). Estrategias y prácticas educativas eficaces para la inclusión educativa: Un estudio de caso en Andalucía. Archivos Analíticos de Políticas Educativas, 18, 1-25,

Florian, L. (2005). "Inclusion", "special needs" and the search for new understandings. Support for Learning, 20(2), 96-98.

Fullan, M. (2007). Las fuerzas del cambio, con creces. Madrid: Akal.

García, C. (2005). Educación y diversidad. Málaga: Ediciones Aljibe. 
Gardou, C., \& Develay, M. (2005). O que as situações de deficiência e a educação inclusiva "dizem" às Ciências da Educação. Revista Lusófona de Educação, 6, 31-45.

González Manjón, D. (2013). La atención a la diversidad en la LOMCE. In C. Marchena González (Dir.), La LOMCE - Claves para el profesorado (pp. 99-122). Madrid: Grupo Anaya.

Instituto Universitario de Integración en la Comunidad (INICO) (2009). Mejor educación para todos: Cuando se nos incluya también. Un informe Mundial. Salamanca: Universidad de Salamanca. Versión electrónica: http://inico.usal.es/inclusion09/ informe.pdf

Jiménez, M., Luengo, J., \& Tabernero, J. (2009). Exclusión social y exclusión educativa como fracasos. Conceptos y líneas para su comprensión e investigación. Profesorado -Revista de Currículum y Formación del Profesorado, 13(3), 11-49.

Khochen, M., \& Radford, J. (2012). Attitudes of teachers and headteachers towards inclusion en Lebanon. International Journal of Inclusive Education, 16(2), 139153.

Kim, J. (2011). Influence of teacher preparation programmes on preservice teachers attitudes toward inclusion. International Journal of Inclusive Education, 15(3), 355-377.

Leitão, F. (2006). Aprendizagem cooperativa e inclusão. Cacém: Editor Ramos Leitão.

Low, C. (2007). A defense of moderate inclusion and the end of ideology. In R. Cigman (Ed.), Included or excluded? (pp. 3-15). London: Routledge.

Marchesi, A. (2000). Controversias en la educación española. Madrid: Alianza Editorial.

Marchesi, A. (2014). Retos y dilemas de la inclusión educativa. In A. Marchesi, R. Blanco, \& L. Hernández (Coords.), Avances y desafíos de la educación inclusiva en Iberoamérica (pp. 37-45). Madrid: Organización de Estados Iberoamericanos (EOI).

Martínez, B. (2005). Las medidas de respuesta a la diversidad: Posibilidades y límites para la inclusión social y la inclusión educativa. Revista Interuniversitaria de Formación del Profesorado, 29 (1), 34-56.

Moliner, O., Sales, M. A., Ferrández, R., Moliner, L., \& Roig, R. (2012). Las medidas específicas de atención a la diversidad en la educación secundaria obligatoria (ESO) desde las percepciones de los agentes implicados. Revista de Educación, 358, 197-217. doi: 10.4438/1988-592X-RE-2011-358-075

Moriña, A. (2011). Aprendizaje cooperativo para una educación inclusiva: Desarrollo del programa PAC en un aula de Educación Primaria. Estudios sobre Educación, $21,199-216$.

Nilholm, C. (2006). Special education, inclusión and democracy. European Journal of Special Needs Education, 21(4), 431-446.

Núñez, M. T. (2008). Educación Especial: Normalizar o volver patológicas as diferenzas? EDUGA -Revista Galega do Ensino, 52, 20-23.

Parrilla, A. (2001). O longo camiño cara a inclusión. Revista Galega do Ensino, 32, 3554. 
Parrilla, A. (2009). ¿Y si la investigación sobre inclusión no fuera inclusiva? Reflexiones desde una investigación biográfico-narrativa. Revista de Educación, 349, 101117.

Pérez, G., \& Sarrate, M. L. (2013). Diversidad cultural y ciudadanía. Hacia una educación superior inclusiva. Educación XXI, 16(1), 85-104.

Pino, M., Domínguez, J., \& López, A. (2007). Evaluating appreciation of measures attending to pupil diversity (EMAD). Psychological Reports, 100, 783-786.

Renold, E. (2000). "Coming out": Gender, (hetero) sexuality and the primary school. Gender and Education, 12(3), 309-326.

Sanches, I. (2005). Compreender, agir, mudar, incluir. Da investigação-acção è educação inclusiva. Revista Lusófona de Educação, 5, 127-142.

Sanches, I. (2011). Do aprender para fazer ao aprender fazendo: As práticas de educação inclusiva na escola. Revista Lusófona de Educação, 19, 157-163.

Sanches, I., \& Teodoro, A. (2006). Da integração à inclusão escolar: Cruzando perspectivas e conceitos. Revista Lusófona de Educação, 8, 63-83.

Shevlin, M., Winter, E., \& Flynn, P. (2013). Developing inclusive practice: Teacher perceptions of opportunities and constraints in the Republic of Ireland. International Journal of Inclusive Education, 17(10), 1119-1133.

Silva, M. O. E. (2003). A análise de necessidades de formação na formação contínua de professores: Um contributo para a integração e inclusão dos alunos com NEE no ensino regular. In R. Baumel (Org.), Educação Especial: Do querer ao fazer (pp. 53-69). São Paulo: Avercamp.

Silva, M. O. E. (2009). Da exclusão à inclusão: Concepções e práticas. Revista Lusófona de Educação, 13, 135-153.

Tavares, C., \& Sanches, I. (2011). Gerir a diversidade: Contributos da aprendizagem cooperativa para a construção de salas de aulas inclusivas. Revista Portuguesa de Educação, 26(1), 307-347.

UNESCO (2004). Temario abierto sobre educación inclusiva. Materiales de apoyo para responsables de políticas educativas. Paris: UNESCO.

Vega, A. (2007). De la dependencia a la autonomía: ¿Dónde queda la educación? Educación XX1, 10, 239-264.

Verdugo, M. A. (2003). De la segregación a la inclusión. Salamanca: Universidad de Salamanca.

Vlachou, A. D. (1999). Caminos hacia una educación inclusiva. Madrid: La Muralla.

Walmsley, J. (2004). Involving users with learning difficulties in health improvement: Lessons from inclusive learning disability research. Nursing Inquiry, 11(1), 5464. 


\title{
Legislación
}

Decreto-lei 3/2008 de 7 de Janeiro. Ministério da Educação.

Decreto-lei 319/91 de 23 de Agosto. Ministério da Educação.

Ley Orgánica 8/2013 para la Mejora de la Calidad Educativa (BOE n. 295, 10/12/2013).

\section{INTEGRAÇÃO OU INCLUSÃO: O DILEMA EDUCATIVO NA ATENÇÃO À DIVERSIDADE}

\begin{abstract}
Resumo
O sistema educativo espanhol advoga a inclusão educativa na atenção à diversidade dos alunos nos centros de ensino obrigatório. Realizou-se uma investigação qualitativa-quantitativa, cujo objetivo era comprovar a substituição paulatina da perspetiva integradora pelas práticas inclusivas nos centros educativos. Assim, os métodos foram implementados de maneira sequencial: numa primera etapa recolheram-se e analisaram-se os dados qualitativos (grupos de enfoque) e, numa segunda fase, recolheram-se e examinaram-se dados quantitativos (inquérito de opinião). A primeira análise mostra um predomínio de aspetos que dificultam a atenção à diversidade (64\%), face aos que a favorecem (36\%). A segunda reflete um predomínio do modelo de integração (74\%), em detrimento do modelo de inclusão (26\%). Além disso, a análise inferencial revela que quanto maior for a experiência docente, menor é a atenção concedida à diversidade, e vice-versa.
\end{abstract}

Palavras-chave

Educação inclusiva; Integração; Atenção à diversidade 
INTEGRATION OR INCLUSION: THE EDUCATIONAL DILEMMA IN ATTENDING TO DIVERSITY

Abstract

The Spanish educational system defends an inclusive approach in attending to the diversity of students in compulsory educational institutions. A qualitativequantitative research was carried out to check the gradual substitution of the integrative practices by the inclusive ones in educational centres. Two methodological phases were completed: in a first step qualitative data were collected and analysed (focus group), and in a second step a quantitative analysis was made (questionnaire feedback). The first analysis (qualitative) shows a predominance of aspects that hinder the attention to diversity (64\%), compared to those which promote it (36\%). The data obtained from the quantitative analysis reflects more integrative tendencies $(74 \%)$, than inclusive ones $(26 \%)$. Also, the inferential analysis indicates that the more educational experience, the less importance is given to the attention to diversity, and vice versa.

Keywords

Inclusive education; Integration; Attention to diversity

Recebido em dezembro/2014

Aceite para publicação em agosto/2015

i Departamento de Análisis e Intervención Psicosocioeducativa (AIPSE), Facultade de Ciencias da Educación, Universidad de Vigo, España.

ii Departamento de Análisis e Intervención Psicosocioeducativa (AIPSE), Facultade de Ciencias da Educación, Universidad de Vigo, España.

iii Departamento de Didáctica, Organización Escolar e Métodos de Investigación, Facultade de Ciencias da Educación e do Deporte (Pontevedra), España.

iv Centro de Formación e Recursos (Ourense), Consellería de Cultura, Educación e Ordenación Universitaria (Xunta de Galicia), España.

Toda a correspondência relativa a este artigo deve ser enviada para: José Domínguez Alonso, Plaza Eduardo Barreiros, n 4 - 2 B, 32003 Ourense, España. E-mail: jdalonso@uvigo.es 